\title{
Auf dem Weg zu einer wirklich allgemeinen Psychologie
}

\section{Reiner Seidel}

\author{
Journal für Psychologie, 26(1), 97-122 \\ https://doi.org/10.30820/8247.06 \\ www.journal-fuer-psychologie.de
}

\section{Zusammenfassung}

In diesem Artikel wird gegen die herkömmliche »Allgemeine Psychologie« der Vorwurf erhoben, dass sie im Wesentlichen eine Psychologie der (höheren und niederen) Tiere ausarbeite, während sie die für den Menschen spezifischen Eigenschaften ignoriere. Kritisiert wird hier weder, dass die herkömmliche Allgemeine Psychologie sich auf Tierexperimente stützt, noch, dass sie mit menschlichen Versuchspersonen arbeitet - beides ist für jede empirische Psychologie unerlässlich. Der Vorwurf geht dahin, dass ihre bei Menschen durchgeführten Experimente meist bei entsprechenden Adaptationen ebenso an Tieren durchführbar sind oder wären. Paradigmatisch hierfür sind bereits die am Beginn der Experimentalpsychologie stehenden Experimente von Ebbinghaus mit sinnlosen Silben, die über 50 Jahre hinweg die Gedächtnisforschung geprägt haben.

Als Alternative wird vorgeschlagen, die Allgemeine Psychologie nach drei »psychischen Stufen « (unbewusst, mental, menschlich) aufzubauen. Diese Allgemeine Psychologie würde - insofern der Mensch psychisch ein Tier ist - all das enthalten, was allen Menschen gemeinsam ist. Hierfür wiederum muss die Psychologie die abgrundtiefe Differenz zwischen Mensch und Tier anerkennen. Der Allgemeinen Psychologie wäre eine »Historisch - kulturelle Psychologie « an die Seite zu stellen, die die konkreten Ausformungen der menschlichen Psyche nachzeichnet.

Der Vorwurf der Vernachlässigung der Mensch-Spezifik wird an fünf Bereichen der Allgemeinen Psychologie verdeutlicht: WAHRnehmung, Gedëchtnis, EMotion, InTELliGENZ, LERNEN.

Das herkömmliche Teilfach Allgemeine Psychologie eröffnet tiefe Einblicke in die tierischen Wurzeln unserer Psyche. Solange diese Allgemeine Psychologie aber die Augen davor verschließt, dass zwischen Mensch und Tier ein Abgrund klafft, kann sie nicht den Anspruch erheben, wirklich allgemein zu sein.

Schlagwörter: Differenz von Mensch und Tier, Grundansätze der Psychologie, Allgemeine versus Historische Psychologie, Spaltung der Psychologie in »naturwissenschaftlich « versus »geisteswissenschaftlich «, Positivismus 


\section{Summary}

Towards a truly general psychology

This article claims that traditional general psychology to a large part develops a psychology of (higher and lower) animals and ignores human specifics. This criticism is not directed at the animal experiments general psychology relies on, nor the experiments with humans - instead both these approaches seem necessary for an empirical psychology. The criticism is that the experiments conducted with humans could - with minor adaptions - also be conducted with animals. The very early experiments of Ebbinghaus with meaningless syllables that have defined memory research for 50 years are paradigmatic.

As an alternative, this article suggests to construct a general psychology based on three psychic levels (unconscious, mental, human). Such a general psychology would include everything all humans share. To achieve this, psychology needs to accept the fundamental difference of humans and animals. General Psychology needs to be aided by a historical-cultural psychology which would be responsible to describe the human psyche.

The claim that the specifically human has been neglected will be exemplified along the lines of five areas: perception, memory, emotion, intelligence, learning.

The traditional sub-discipline general psychology allows for a thorough examination of the animalistic roots of our psyche. But as long as such a general psychology ignores the difference of animals and humans, it cannot really be called > general<.

Keywords: difference of humans and animals, foundational approaches to psychology, general versus historical psychology, split of natural science and humanities, positivism

\section{Terminologische Vorbemerkung}

In dem Ausdruck »allgemeine Psychologie « (kleingeschrieben) ist das Adjektiv umgangssprachlich gemeint. In diesem Artikel geht es um das Standard-Fachgebiet innerhalb der Wissenschaft Psychologie, hier wird das Adjektiv großgeschrieben: »Allgemeine Psychologie « und mit » $\mathrm{AP} \ll$ abgekürzt.

\section{Was heißt »allgemeine Psychologie«?}

Nach den ersten Entwürfen rationalistischer (R. Descartes) und empiristischer (J. Locke) Psychologie beziehungsweise Philosophie im 17. Jahrhundert hatte sich die Psychologie soweit differenziert, dass um 1800 die Unterscheidung zwischen allgemeiner und spezieller Psychologie aufkam (Eckardt et al., 2001). 
»In der allgemeinen Seelenlehre (Psychologia generalis) werden diejenigen Merkmale und Naturgesetze des menschlichen Geistes betrachtet, die allen Individuen des Menschengeschlechtes immer und ohne Ausnahme zukommen oder zukommen können. Die besondere Seelenlehre (Psychologia specialis) untersucht die Verschiedenheit, wodurch sich die einzelnen Menschenklassen unterscheiden «(Schmid, 1796, zit. n. Schönpflug, 2000, S. 220).

Die »spezielle« Psychologie hat sich später, je nach dem von den Autoren gewählten Grundansatz, als »Charakterologie « oder »differenzielle Psychologie « etabliert.

Auch nach heute allgemein akzeptierter Auffassung betrachtet die »Allgemeine Psychologie $\ll(A P)$

»den Menschen als psychologisches Gattungswesen und fragt nach dem, was Menschen gemeinsam ist - ohne sich dafür zu interessieren, was sie unterscheidet « (Müsseler \& Prinz, 2002, S. 4).

Von der Unterscheidung »Allgemeine « gegenüber »Differenzielle « Psychologie gehe auch ich aus, stelle aber die »Allgemeinheit « der traditionellen AP infrage.

Zuallererst ist zu fragen, was man unter der Psyche, dem Gegenstand der PsychoLogie, verstehen will. Soweit ich sehe, ist es heute akzeptabel, »Psyche « als »Steuerung des Verhaltens « zu begreifen. Der Bezug auf »Verhalten « impliziert den Bezug auf das Reich der Tiere.

Die psychischen Eigenschaften der Tiere (Menschen eingeschlossen) beruhen auf den allgemeinen Eigenschaften der Lebewesen, zu denen auch die Pflanzen gehören. Insofern fundieren auch die anatomischen und physiologischen Funktionen des Menschen seine Psyche. Da man Pflanzen schlecht »Verhalten «zuschreiben kann, bezeichne ich das Gebiet der vegetativen Funktionen als »Prä-Psyche«.

Was aber ist das » Gattungswesen « Mensch? Was ist allen Menschen gemeinsam? Was kommt dem Menschen von Natur aus zu? Seit sich ab Mitte des 19. Jahrhunderts die Evolutionstheorie durchsetzte, ist für aufgeklärte Menschen klar, dass der Mensch sich aus dem Tierreich heraus entwickelt hat. Und zwar ist der Mensch nicht nur anatomisch und physiologisch, sondern und gerade auch in seiner Psyche tierischen Ursprungs. »Der Mensch von Natur aus « kann also nur heißen: »Der Mensch als Tier «.

\section{Mein Desiderat:Psychologie auf zwei Standbeinen:»Allgemeine Psychologie» und "Historisch-kulturelle Psychologie»}

Wenn das Fachgebiet AP die (psychischen) Gattungsmerkmale des Menschen - jenseits individueller oder historisch-kultureller Unterschiede - beschreiben soll, und wenn der 
Mensch aus dem Tierreich entstanden ist, dann liegt auf der Hand: die AP sollte den Unterschied von Mensch und Tier zum Ausgangspunkt nehmen. Diesem Vorsatz kann man meines Erachtens kaum widersprechen, aber nun ergibt sich die Frage: Kann man die Psyche des Menschen von der Psyche der Tiere wirklich unterscheiden, und wenn ja, wie kann man sie im Einzelnen unterscheiden?

Leider ist diese Schlüsselfrage in Philosophie und Wissenschaft höchst umstritten, es gibt sogar völlig konträre Positionen. Auf der einen Seite steht die Auffassung, es gebe Merkmale (etwa Sprache und Denken), die nur Menschen haben, aber kein einziges Tier. Verallgemeinert kann man dies als Alleinstellungsthese bezeichnen. Aus dieser These folgt, dass zwischen Mensch und Tier letztlich ein unüberbrückbarer Abgrund besteht. Auf der anderen Seite steht die konträre Auffassung: Die Menschen sind aus dem Tierreich entstanden, die menschliche Psyche ist daher »aus dem gleichen Stoff gemacht « wie die der Tiere. Dies möchte ich als Gemeinsamkeitsthese bezeichnen.

\subsection{Die Alleinstellungsthese und das Konzept der Ebenen (psychische Stufen)}

Nach meiner Konzeption ist die Psychologie auf der Basis »psychischer Stufen « nach der Evolution aufzubauen: unbewusst, mental, menschlich. Der Ansatz basiert auf der Theorie von Leontjew und der Kritischen Psychologie (Holzkamp), unterscheidet sich hiervon aber in wesentlichen Punkten. Den Leser des vorliegenden Artikels bitte ich um Nachsicht, dass ich meinen Ansatz hier nicht ausführlich darlegen kann und hierzu auf mein im Druck befindliches Buch Die Evolution der Psyche. Wieviel Tier ist der Mensch? (Seidel, 2018) verweisen muss. Der hiesige Artikel konzentriert sich auf den Begriff »Allgemeine Psychologie «. Hier möchte ich nur kurz angeben, worauf nach meiner Auffassung die Alleinstellung des Menschen mit allen ihren Folgen letztlich beruht: Das menschliche Gehirn - im Unterschied zu den Gehirnen aller anderen Tiere (einschließlich der Affen) - setzt seinen Träger in die Lage, die Welt als Außenwelt aufzufassen. Ich fasse diesen spezifisch menschlichen »Glauben an die Außenwelt « als Fähigkeit zur »Reflektion «.

Aus der Alleinstellung folgt insbesondere, dass der Mensch, im Unterschied zu den Tieren, Geschichte und Kultur hat. Im vorliegenden Artikel muss ich auch diesen Ausgangspunkt schlicht als Behauptung hinsetzen. Der Mensch ist in seinem Wesen, das heißt von vornherein, sobald er als homo sapiens in der Evolution auftaucht, historisch, das heißt er nimmt bewusst Einfluss auf seine Lebensbedingungen. Insoweit dieses Einflussnehmen sich nach unterschiedlichen Formen differenziert, ist von Kultur zu sprechen. Andererseits gilt, dass beim Tier weder Geschichte noch Kultur zu finden ist.

Seit geraumer Zeit gibt es eine breit gefächerte engagierte Literatur gegen die Al- 
leinstellungsthese. So vertreten die Sprachtrainer des Bonobo »Kanzi«, dem man den Gebrauch von Zeichen mittels an einer tragbaren Tafel angebrachter Bilder beibringen konnte, dass es keinen grundsätzlichen Unterschied zwischen menschlicher Sprache und tierischer Kommunikation gebe:

»What Kanzi tells us is that humans are not the only species that can acquire language [...] Humans simply appear to be the only species that can make the proper sounds « (SavageRumbaugh et al., 1998, S. 74).

Dass es Ähnlichkeiten bei Mensch und Tier gibt, ist aufgrund der Evolution trivial. Offenbar sehen viele Menschen die Alleinstellungsthese und die Gemeinsamkeitsthese als logischen Widerspruch. Ich habe nie verstanden, worin dieser Widerspruch bestehen soll, ich denke eher, diese vermeintliche »Logik « ist falsch, wie ich im Folgenden kurz begründen will.

Die Gegner der Alleinstellungsthese sehen sich - fälschlicherweise, wie ich meine - durch die Evolutionstheorie gestützt. Darwins Theorie hat die gesamte Lebens-Wissenschaft vor allem dadurch revolutioniert, dass sie die Entstehung der Arten - die Art »Mensch « eingeschlossen - aus einer Abfolge kleiner und kleinster Schritte erklärt und so den Schöpfungsglauben ersetzt. Auch ich bin fest davon überzeugt, dass das Neue durch schrittweise kleine Veränderungen zustande kommt. Ebenso bin ich aber überzeugt, dass in der Abfolge kleiner Schritte, wenn auch vergleichsweise selten, besondere Veränderungen auftreten, die ich als »konstitutive Veränderungen « bezeichnen will, das heißt als Veränderungen, die - abgesehen von ihrem Effekt der Entstehung einer speziellen Spezies - sich auf die Bedingungen der Evolution selbst auswirken. Wenn man so will, kann man von »neuen Qualitäten «, »qualitativen Sprüngen «, »Quantensprüngen « oder auch von »Revolutionen in der Evolution « sprechen. Eine »Revolution « dieser Art stellt zum Beispiel der Übergang von den Pflanzen zu den Tieren dar. Ich verstehe nicht, warum nicht auch der Übergang vom Tier zum Menschen, genauer: die Entstehung des spezifisch menschlichen Gehirns, als eine solche Revolution akzeptiert wird.

Eine beliebte Formulierung in der Problematik des Tier/Mensch - Verhältnisses besteht darin, dass man Unterschiede von Mensch und Tier im Sinne des Positivismus als » rein quantitativ « erklärt. Beispielsweise beherrsche der Mensch, was die Sprache betrifft, zwar mehr Zeichen als der Affe, könne sie schneller verarbeiten, sei flexibler in der Informationsverarbeitung usw. Dies alles sei nur eine Frage von Mehr oder Weniger, in der Qualität liefen menschliche und äffische Sprache auf dasselbe hinaus. Ich sehe in dieser Art Argumentation einen bequemen Vorwand, sich nicht tiefer in das Wesen der Sprache einzulassen. Man ignoriert beispielsweise schlicht die »Darstellungsfunktion «, das heißt, dass die menschliche Sprache, zusätzlich zum Zeichenaustausch zwischen 
Sender und Empfänger, sich auf Gegenstände bezieht (Bühler, 1934). Ebenso ignoriert man die nachweisbare Unfähigkeit der Tiere zur Grammatik (u. a. Pinker, 1994/1996). Ich werde nachher noch auf weitere Simplifizierungen dieser Art zu sprechen kommen.

\subsection{Methodik: Empirie und Rekonstruktion}

Ich setze voraus: Psychologie ist eine empirische Wissenschaft, die sich auf Beobachtung und Experiment stützt. Der Bezug auf die Empirie wirft zugleich ein Problem auf. Wenn der Mensch als Gattungswesen von Anfang an historisch und kulturell verfasst ist, müsste die AP des Menschen nicht alle geschichtlichen Etappen und die diversen kulturellen Formen der psychischen Phänomene einbeziehen? Offenbar geriete so die $\mathrm{AP}$ zu einem unendlichen Projekt. Denkmöglich sind zwei Auswege in diesem Dilemma. Erstens könnte man den Anfang der Menschheit, mithin den Urmenschen, zum Startpunkt nehmen. Die Anthropologie geht davon aus, dass homo sapiens nur einmal, und zwar in Ostafrika, entstanden ist, es muss den Urmenschen also einmal gegeben haben, aber mehr als ein paar Knochen und Spuren haben wir nicht von ihm. Für eine empirische und mithin beschreibende Wissenschaft wie die Psychologie ist dieser Ausweg also nicht gangbar, denn wir können das Verhalten des Urmenschen nicht beobachten.

Was als Ausweg verbleibt, ist, zweitens, die Rekonstruktion. Das heißt, wir gehen aus vom (direkt beobachteten oder tradierten) Verhalten des Menschen und fragen rückwirkend, welche psychischen Prozesse erforderlich sind oder waren, um dieses Verhalten oder diese Leistungen zu bewirken. Letztlich liegt Rekonstruktion dieser Art aller AP zugrunde.

Um nicht ins Uferlose zu geraten, muss man sich dabei an das bis ins 14. Jahrhundert zurückgehende und weithin akzeptierte Sparsamkeitsprinzip halten: Wenn mehrere Möglichkeiten der Erklärung einer Erscheinung bestehen, dann soll man die einfachste Theorie wählen, das heißt diejenige, die mit den geringsten Voraussetzungen auskommt.

\subsection{Konzept einer wirklich allgemeinen Psychologie}

Nach dem eben vorgetragenen Gedankengang bietet sich folgender Aufbau der Wissenschaft Psychologie an. Grundlage ist eine »Allgemeine Psychologie «(AP). Sie umfasst alles, was wir über die Psyche sagen können. Dies sind zum einen die vegetativen Voraussetzungen der Psyche - ich fasse diese, wie gesagt, als »Prä-Psyche «. Zweitens bestehen die allgemeinsten psychischen Eigenschaften in unserem tierischen Erbe. Ich fasse dies als unsere »animalische Psyche « zusammen. 
Genauer sollte die » animalische Psyche « noch einmal unterschieden werden nach a) einer » unbewussten « Psyche, das heißt einer Verhaltenssteuerung, die ohne b) das Mentale (Erleben, Bewusstsein, Empfindung) auskommt. Tiere, für die nur einfache Reaktionen im Sinne von a) ausreichen, bezeichne ich als »niedere Tiere «, solche die darüber hinaus mentale Prozesse aufweisen, bezeichne ich als » höhere Tiere «. Für den vorliegenden Artikel lasse ich diese Unterscheidung beiseite.

Drittens schließlich soll die AP die allgemeinen Eigenschaften des Menschen darstellen. Hierbei ist zu berücksichtigen, dass sich beim Menschen - nur bei ihm - ein zweiter Zweig öffnet, die Historisch-kulturelle Psychologie. Sie zeichnet die konkreten Ausgestaltungen der Psyche auf, wie sie sich in den historischen Epochen und in den diversen Kulturen äußern. Der Unterschied von der AP des Menschen einerseits und der Historisch-kulturellen Psychologie andererseits wird oft übersehen. Ja, manche Vertreter einer sogenannt » naturwissenschaftlichen Psychologie « neigen dazu, die AP des Menschen abzuschieben in die Historisch-kulturelle Psychologie. Das ist nach meinem Konzept aber grundfalsch. Denn es gibt grundlegende für den Menschen spezifische Merkmale über alle historisch-kulturellen Ausgestaltungen hinweg wie Sprache, Kunst, Religion usw. In Abschnitt 3 werde ich das Ignorieren solcher Merkmale in Form von Mangelanzeigen über die ganze Breite der Psyche hinweg aufzeigen.

Der Ansatz ist in Tabelle 1 zusammengefasst. Die Unterteilung in der Spalte Historisch-kulturelle Psychologie sind nur als Illustration von Möglichkeiten gedacht (dass ich als Angelpunkt die Zivilisation nenne, ist hier nur als Illustration gemeint; wahrscheinlich werden die Kultur-Varianten für die Epoche der Zivilisation reichhaltiger sein als die vor der Zivilisation).

\begin{tabular}{|c|c|c|}
\hline Allgemeine Psychologie & \multicolumn{2}{|c|}{ Historisch-kulturelle Psychologie (Mensch) } \\
\hline \multirow{6}{*}{ Mensch-Psyche } & \multirow{3}{*}{ seit Zivilisation } & Kultur xy \\
\hline & & Kultur xy \\
\hline & & Kultur xy \\
\hline & \multirow{3}{*}{ vor Zivilisation } & Kultur xy \\
\hline & & Kultur xy \\
\hline & & Kultur xy \\
\hline mentale Psyche & & \\
\hline unbewusste Psyche & & \\
\hline Prä-Psyche & & \\
\hline
\end{tabular}

Tab. 1: Basaler Aufbau der Psychologie 
Das in Tabelle 1 angegebene Schema ist dazu gedacht, auf jeden einzelnen Bereich (Kognition, Emotion, Lernen usw.) bezogen zu werden. Man beachte, dass die »Allgemeine Psychologie « aus drei Ebenen zusammengesetzt ist, im Unterschied zur Historisch-kulturellen Psychologie, die sich nur auf den Menschen bezieht.

\section{These: Die traditionelle AP ignoriert den Unterschied zwischen Mensch und Tier}

Mit diesem Abschnitt stelle ich eine Versäumnisliste der gegenwärtigen AP auf.

\subsection{Mangelanzeige aus dem Bereich WAHRnehmung}

Eines der Flaggschiffe der AP sind die Sinnestäuschungen wie die bekannten optischen Täuschungen, an denen insbesondere die Gestaltpsychologie ihre Freude findet. Sie sind populär, unter anderem da sie effektvoll bei Menschen demonstriert werden können. In der Regel wird dabei übersehen, dass sie ein animalisches, keineswegs nur ein mensch-spezifisches Phänomen sind. So ist gezeigt worden, dass bereits Fische, Vögel und Säugetiere solcherart Täuschungen unterliegen (vgl. z. B. Rensch, 1973). Dass die optischen Täuschungen in der Wahrnehmungspsychologie großen Raum einnehmen, ist kein Fehler, denn sie sagen Wesentliches über die Organisation des Wahrnehmens aus. Gleichwohl ist ihre prominente Stellung in der AP ein Beleg meiner These, dass die AP wesentlich eine Psychologie höherer Tiere sei.

\subsection{Mangelanzeige aus dem Bereich GeDÄCHTNIS}

Besonders aufschlussreich ist die frühe Experimentalpsychologie, in der H. Ebbinghaus der Psychologie für Jahrzehnte neue Impulse gegeben hatte. Dieser Pionier hatte über Jahre Listen von »sinnlosen Silben « auswendig gelernt und seine Behaltensleistung systematisch ausgewertet. Sinnlose Silben hatte er eigens konstruiert, um den Einfluss früherer Assoziationen auszuschalten (Ebbinghaus, 1885). Ebbinghaus' Ziel war es, möglichst nahe an das »natürliche Gedächtnis « heranzukommen. Durch die Sinnlosigkeit der Silben hatte er den Einfluss von Sprache und Kultur eliminiert. Die unvermeidliche Variabilität der Versuchsbedingungen, später auch die Verschiedenheit individueller Versuchspersonen, glich er über statistische Methoden aus. Nicht im Traum konnten Ebbinghaus und Zeitgenossen sich damals vorstellen, dass man heutzutage sogar neuronale Schaltkreise des Gedächtnisses beschreiben kann, wie 
es zum Beispiel bei der Meeresschnecke Aplysia gelungen ist (z. B. Kandel, 2006). Mit Sicherheit würde Ebbinghaus heutzutage neuropsychologisch arbeiten und seine Ergebnisse in einem der Journale der Neurobiologie, Biopsychologie oder der AP veröffentlichen.

Kann man die Gedächtnisleistungen eines Menschen beim Auswendiglernen von Silben mit den Reaktionen einer in einer Petrischale liegenden Schnecke vergleichen? Selbstverständlich kann man das, ebenso wie man den physiologischen Grundumsatz eines grübelnden Mathematikers messen kann. Es hängt davon ab, auf welcher (psychischen) Ebene sich die Forschung bewegt. Von einer Ratte beispielsweise wird man auf den ersten Blick nicht direkt verlangen können, sie solle Silben auswendig lernen. Wenn man ihr jedoch Möglichkeiten anbietet, die ihrer körperlichen Ausstattung entsprechen, kann sie sehr wohl Vergleichbares leisten. Wie die Lerntheorie gezeigt hat, kann man zum Beispiel eine Ratte auf mehrstufige Verhaltensketten konditionieren, sodass sie etwa nacheinander Leitern auf verschiedenen Sprossen erklimmt. Nehmen wir also statt fünf sinnloser Silben fünf verschiedene kleine Flaggen, die wir an verschiedenen Stellen des Raums aufstellen. Durch geeignete Verstärkung kann man die Ratte darauf konditionieren, dass sie die Flaggen in einer bestimmten Reihenfolge aufsucht. Rein als Gedächtnisleistung hat sie somit ihre fünf $\gg$ Silben « auswendig gelernt. Wenn man so will, kann man anschließend in den Fußstapfen von Ebbinghaus das Rattengedächtnis weiter beforschen. Man könnte untersuchen: das Behalten als Funktion der Anzahl der Wiederholungen, das Behalten und Vergessen als Funktion der Zeit, und vieles anderes mehr (Ebbinghaus, 1885).

Zugegeben, bei der Meeresschnecke Aplysia wird man ein derartiges Experiment nicht machen können, da sie wahrscheinlich nicht verschiedene Reize innerhalb derselben Versuchsanordnung unterscheiden und jeweils darauf reagieren kann. Ein Tier wie die Ratte jedoch kann so etwas. Die vorangegangenen Überlegungen sollen klar machen: es kommt nicht darauf an, ob Experimente an Menschen oder Tieren durchgeführt werden, sondern auf welcher psychische Stufe sie sich abspielen.

Man mag einwenden, heutzutage arbeite kein Mensch mehr mit sinnlosen Silben. Wirklich? Mit dem Kognitivismus ab den 1960er Jahren verlagerte sich das Interesse der Psychologie auf die Sprache. Man darf aber nicht glauben, damit sei die AP zur menschlichen Spezifik vorgedrungen. Eben habe ich gezeigt, dass man mit Ratten ein Äquivalent zu sinnlosen Silben herstellen kann. Im Kontext der »kognitiven Wende « hatte der Gedächtnisforscher E. Tulving zu Recht darauf hingewiesen, dass der Mensch neben einem Gedächtnis für die Sprache (»semantisches Gedächtnis «) ein Gedächtnis für persönlich erlebte Inhalte besitze. Damit wäre man in der Tat beim spezifisch Menschlichen, nämlich beim autobiografischen Gedächtnis, angelangt. Für das, was er meinte und das er als » episodisches Gedächtnis « bezeichnete, gibt Tulving folgendes Beispiel: 
$\gg$... one may remember a recent trip to Paris, mentally reliving events that happened there, in the mind's eye seeing again the places visited, sights seen, sounds heard, aromas smelled, and people met $\ll$ (Tulving \& Szpunar, 2009).

Jedoch: was in der Forschung zum »episodischen Gedächtnis « tatsächlich geschieht, hat mit autobiografischem Gedächtnis beziehungsweise Erinnerung nichts, aber auch gar nichts, zu tun. Denn das Basismaterial dieser Experimente besteht in Listen von zusammenhangslosen, zufällig ausgewählten Wörtern. Also: Von den »sinnlosen Silben « zu den $\gg$ sinnlosen Wörtern «! das ist der Fortschritt von Ebbinghaus zu Tulving. Wie kommt Tulving auf die Idee, sein »episodisches Gedächtnis « laufe auf so etwas wie »Erinnerung « oder eben Autobiografie hinaus? Zu Recht führt er zwar an, dass in der Erinnerung das Subjekt persönlich erlebte Dinge und Situationen im Gedächtnis behalte. Jedoch entgeht ihm vollkommen, dass der Mensch gerade das behält, was ihm subjektiv wichtig erscheint. Wenn Versuchspersonen - üblicherweise Psychologie-Studenten - sich an ihre Teilnahme an einem Tulving-Experiment erinnern, werden sie vielleicht daran denken, dass sie ihren Versuchspersonen-Schein erhielten oder dass sie eine interessante Person wahrnahmen oder dass es drückend heiß war. Aber dafür interessieren sich die Experimentatoren überhaupt nicht. Sie fragen nur nach den (sinnlosen) Wortlisten, die den Versuchspersonen wiederum vollkommen gleichgültig sind.

Im Prinzip könnte man das »episodische Gedächtnis «, analog etwa zu meiner hypothetischen Ratte, auch an Ratten oder Affen untersuchen. Man müsste nur vor dem Experiment zwischen den fünf Flaggen Assoziationen stiften - analog zu den zwischen Wörtern bestehenden semantischen Zusammenhängen. Das Manöver von Tulving ist, so gesehen, ein Ettikettenschwindel.

Ein Kümmerdasein gegenüber der kognitivistischen Gedächtnispsychologie fristet dagegen die Forschung zur Erinnerung oder zum autobiografischem Gedächtnis, der spezifisch menschlichen Form des Gedächtnisses. Alles in Allem, der Löwenanteil in der traditionellen AP fällt dem animalischen Gedächtnis zu, das autobiografische Gedächtnis (Erinnerung) wird, wenn überhaupt, behandelt wie eine arme Verwandte.

\subsection{Mangelanzeige aus dem Bereich ЕмотіоN}

Der Bereich der Emotionalität ist in besonderer Weise geeignet, die Aufteilung nach Allgemeiner versus Historisch-kultureller Psychologie (vgl. Tabelle 1) zu verdeutlichen. Seit ihren Anfängen hat sich die AP dieses Bereichs ausführlich um die physiologischen Grundlagen von Gefühlen gekümmert. Man breitet - oft bis ins kleinste Detail - die körperlichen Begleitphänomene aus, wie sie mit Emotionen wie Wut, Trauer, Freude usw. einhergehen. Soweit, so gut. Hierbei würde ich, um den endlosen terminologi- 
schen Wirrwarr zu beenden, den emotionalen Bereich in den neuronalen Schaltkreisen (vgl. Panksepp \& Biven, 2012) verankern.

Heftige Debatten wurden um die Rolle der animalischen Grundlagen gegenüber den kulturellen Erscheinungsformen von Emotionen geführt. Insbesondere unter dem Einfluss des amerikanischen Anthropologen P. Ekman (vgl. u.a. Ekman, 1992) ist auf dem Feld eine gewisse Beruhigung eingetreten. Ekman und andere hatten die Welt bereist und nachgewiesen, dass es sieben bis acht $\gg$ Basis-Emotionen « gibt, die in allen Kulturen ähnlich verstanden werden.

Da Emotionen offensichtlich aber auch kulturell äußerst unterschiedliche Ausdrucksformen haben können, liegt es nahe, ein Gebiet wie »Kulturpsychologie«, »kulturvergleichende Psychologie «, »cross-cultural psychology« usw. einzurichten (vgl. z. B. Trommsdorff \& Birbaumer, 2007). Mein Vorschlag einer »Historisch-kulturellen Psychologie « meint genau dies.

Gleichwohl ignoriert die traditionelle AP, soweit ich sehe, einen generellen Unterschied zwischen menschlichen und animalischen Emotionen, unabhängig von aller Historie und Kultur. Bei den Tieren besteht sozusagen eine 1:1 - Beziehung zwischen Emotionen und den durch die Physiologie nahegelegten Äußerungsformen. Beim Menschen dagegen sind die Äußerungsformen von Emotionen im Prinzip unbegrenzt. Wut zum Beispiel drückt sich zwar standardmäßig in wilden Verhaltensweisen wie Umherschlagen, Brüllen usw. aus und kann am Beispiel eines gereizten Stiers veranschaulicht werden. Bei einem Menschen aber kann starke Wut sich ebenso dadurch äußern, dass er, ohne einen Laut von sich zu geben und ohne mit der Faust auf den Tisch zu schlagen, sich einen Racheplan ausdenkt.

Zwar bauen sich alle menschlichen Emotionen aus den animalischen Emotionen auf, trotzdem haben Emotionen beim Menschen eine grundlegend andere Qualität als bei Tieren. Dies zeigt sich bereits beim körperlichen Schmerz. Natürlich ist es derselbe Schmerz, den ein Hund oder ein Mensch spürt, wenn er ein Bein verliert. Aber phänomenologisch ist das, was der Mensch dabei erlebt, etwas total Anderes. Der Hund spürt den Schmerz körperlich und wird das Hinken als Beeinträchtigung erleben. Für einen Sportler dagegen kann, darüber hinaus, seine ganze Existenz, seine Zukunftsvorstellung zusammenbrechen usw.

Ein weiteres Beispiel ist die Trauer. Wenn etwa einer Affenmutter ein Kind abhanden kommt, läuft sie depressiv herum, verweigert Nahrung oder dergleichen. Richtig, bei Menschen geschieht Ähnliches, trotzdem spielt sich menschliche Trauer in einer anderen Welt ab als bei den Tieren. Bereits die bloße Tatsache, dass Tiere niemals Trauer-Zeremonien veranstalten, beweist dies.

Alles in Allem, die traditionelle AP konzentriert sich weitgehend auf das, was die Emotionalität der Menschen mit der der Tiere gemeinsam hat und geht kaum auf die Spezifik des Menschen ein. 


\subsection{Mangelanzeige aus dem Bereich INTELLIGENZ}

Bei der Intelligenz muss ich auf eine Annahme zu sprechen kommen, von der keineswegs nur die traditionelle AP, sondern ein großer Teil der Anthropologie (fälschlicherweise) ausgeht. Auch in Alltagsgesprächen fällt es manchmal schwer, den Unterschied von menschlichem gegenüber animalischem Werkzeuggebrauch klarzumachen. Im technischen Sinn ist Werkzeuggebrauch bereits bei niederen Tieren und erst recht bei höheren Tieren vorzufinden. Ja, gewisse Vögel, vor allem aber auch Affen, können Gegenstände zum Gebrauch als Werkzeug bearbeiten, können also Werkzeuge sogar herstellen. Aber einzig der Mensch nimmt sich Gegenstände heran mit dem Ziel, diese als Werkzeug zu benutzen. Daher entwickelt auch nur der Mensch seine Werkzeuge weiter.

Schwer verständlich ist für mich, dass die Leugner der Alleinstellung des Menschen den simplen Sachverhalt übersehen, den schon der Gestaltpsychologe Wolfgang Köhler (1917/1921) registrierte: Affen (wie alle anderen werkzeugbenutzenden Tiere) verlieren ihre Werkzeuge nach deren aktuellem Gebrauch aus dem Sinn, heben sie zu einer späteren Verwendung nicht auf (reiner ad-hoc-Werkzeuggebrauch). Ein Kollege meinte einmal zu mir, die Affen würden Steine, mit denen sie Nüsse aufschlagen, nur deshalb nicht aufbewahren, weil Steine überall herumlägen; wenn es nur selten Steine gäbe, würden sie diese aufbewahren. Ich fragte zurück, ob er an den Weihnachtsmann glaube. Werkzeug benutzende Tiere können zwar den »Funktionalwert « (Köhler) von Gegenständen für den Gebrauch als Werkzeug erkennen, aber ihr Gehirn ist damit überfordert, Werkzeuge aus dem Rahmen der zu befriedigenden Bedürfnisse herauszulösen.

Ebenso oberflächlich wie der Begriff des Werkzeugs wird in der traditionellen AP der Begriff der $Z a b l$ benutzt. Seit langem ist bekannt, dass manche höheren Tiere wie Vögel und Säugetiere mit Anzahlen umgehen können. Zum Beispiel kann man eine Ratte darauf konditionieren, dass sie genau fünf Mal einen Hebel betätigt. Oder man kann Hühner dazu bringen einen Deckel mit drei von einem Deckel mit sechs Punkten zu unterscheiden. Affen gelingen sogar Additionen bei kleinen Anzahlen. Wenn man in einschlägigen Publikationen liest, die Versuchstiere Tiere könnten »Zahlen « benutzen oder verstehen, so ist dies nicht weniger schlampig wie der angebliche »Werkzeug «gebrauch. Jedes Schulkind erwirbt relativ schnell einen Begriff der Zahl, der, wie J. Piaget demonstriert hat, operativer Natur ist, das heißt nicht durch bloße Anschauung, sondern über geistige Operationen erzeugt wird.

So wie man beim Werkzeuggebrauch nur hinschauen muss, um festzustellen, dass Tiere ihre Werkzeuge anschließend nicht mehr beachten, so gibt es ein simples Kriterium dafür, ob ein Kind den operativen Zahlbegriff versteht oder nicht. Wenn der Begriff der Zahl operativ vorhanden ist, so ist er unbegrenzt, das heißt das Kind ver- 
steht, dass es immer noch eine höhere Zahl geben wird. Die erwähnten tierischen Leistungen sind dagegen auf die direkte sinnliche Wahrnehmung begrenzt. So hören die erwähnten Leistungen der Tiere schlagartig auf, sobald es um größere Anzahlen geht.

Insgesamt lässt sich festhalten: Die traditionelle AP thematisiert bei der Intelligenz im Wesentlichen Leistungen, die auch anderen Tieren möglich sind (zu einer Ausnahme siehe Abschnitt 4).

\subsection{Mangelanzeige aus dem Bereich LeRNEN}

Als Grundformen des Lernens sieht die traditionelle AP zum einen die »klassische « oder »Pawlowsche « Konditionierung und zum andern die »operante « Konditionierung (Skinner). Dass diese Konditionierungsformen in der traditionellen AP gründlich besprochen werden, ist kein Fehler, sondern eine Notwendigkeit, denn auch das Leben der Menschen ist durch Konditionierungen geprägt. Zu kritisieren ist dagegen, dass die darüber hinausgehenden spezifisch menschlichen Möglichkeiten des Lernens weitgehend undiskutiert bleiben. Ich nenne drei Aspekte:

a) Bei dem grundlegenden Prinzip der (positiven oder negativen) Verstärkung gibt es einen theoretisch bedeutsamen Unterschied. Bei Tieren lassen sich die möglichen Verstärker an einer Hand abzählen (Futter oder Streicheln, das man gibt oder entzieht). Beim Menschen dagegen kommen Behavioristen in Schwierigkeiten, denn beim Menschen kann prinzipiell alles als Verstärker beziehungsweise als Strafreiz dienen. Eine Theorie, die dies übersieht, muss in unlösbare Schwierigkeiten kommen.

b) Konditionierungen sind bei Tieren immer nur Begleitvorgänge der jeweiligen Tätigkeit. Keinem Tier wird es je einfallen, eine Aktion deshalb noch einmal stattfinden zu lassen, damit sie beim nächsten Mal besser gelingt. Solches »intentionale « Üben oder Lernen spielt beim Menschen dagegen eine fundamentale Rolle.

c) Menschliches Lernen kann darauf abzielen, die Sache, um die es geht, zu verstehen. Die grundsätzliche Bedeutung dieser Intention wird zwar oft vernachlässigt, ist aber immerhin möglich und sogar anstrebenswert.

Schließlich soll folgendes Beispiel zeigen, dass die Beschränkung auf den Horizont animalischen Lernens praktische Auswirkungen auf pädagogisches Verhalten haben kann: Lehrer sind verärgert, wenn Schüler beim Gong schlagartig ihre Sachen zusammenpacken, anstatt das Ende der Stunde abzuwarten. Es ist sicher nicht falsch, wenn solches Schülerverhalten durch »Konditionierung « erklärt wird. Meinethalben 
mag eine pädagogische Anleitung für Lehrer auch Vorschläge zur Umkonditionierung erwägen. Aber ist das alles? Bezieht man die spezifisch menschlichen Bedingungen und Möglichkeiten des Lernens ein, so wird man feststellen, dass Schüler (außer ihrer Konditionierbarkeit) vor allem zur Reflektion fähig sind. Man könnte zum Beispiel das fragliche Verhalten zum Gegenstand einer Diskussion machen. Ist den Schülern überhaupt klar, dass sie implizit den Lehrer beleidigen? Oder umgekehrt: Ist das vorschnelle Einpacken vielleicht als stummer Protest gegen einen langweiligen Unterricht zu werten?

Alles in Allem, die traditionelle AP konzentriert sich auf Lernvorgänge, die sich bereits bei Tieren finden.

\section{Nur ausnahmsweise thematisiert die traditionelle AP spezifisch menschliche Phänomene}

Ich glaube gezeigt zu haben, dass die traditionelle AP im Wesentlichen von der Psyche höherer Tiere - zu denen ja auch der Mensch gehört - handelt und das, was darüber hinaus geht, kaum berührt. Einige wenige Ausnahmen gibt es, vor allem im Bereich INTELLIGENZ. Ich meine das »logische Denken «, denn hier kommen standardmäßig auch Dinge zur Sprache, die nach meiner Systematik in die Sparte »Historisch-kulturelle Psychologie « gehören. Denn das logische Denken, insofern man es mit Mathematik oder symbolischer Logik verbindet, ist eine Erscheinung, die es erst seit der Zivilisation gibt. Dass es dennoch in der traditionellen AP gelandet ist, liegt vermutlich an der umfassenden Bedeutung, die Mathematik und Logik heute in Wissenschaft und Technik besitzen.

\section{$5 \quad$ Fazit}

Der Mensch stammt nicht nur im weiten Sinn biologisch, sondern und gerade in seiner Psyche aus dem Tierreich. Daher muss die Psychologie an der Psyche der niederen und höheren Tiere ansetzen, wobei sie zudem die Basis des Vegetativen (»Prä-Psyche «) einzubeziehen hat. Das Fachgebiet »Allgemeine Psychologie « (AP) übernimmt diese Aufgabe. Das ist gut so. Nicht gut ist dagegen, dass die traditionelle AP im Wesentlichen bei der animalischen Psyche stehen geblieben ist und somit das eigentliche Ziel, die Psyche des Menschen, verfehlt. In diesem Sinne ist die traditionelle AP nicht allgemein. Da man mit Recht von einer Psychologie erwartet, dass sie sich mit dem Menschen befasst, erscheint die traditionelle AP als wenig relevant, kleinkariert und langweilig. 


\section{Literatur}

Bergson, H. (1908 [1896]). Materie und Gedächtnis. Essays zur Beziehung zwischen Körper und Geist. Jena: Eugen Diederichs.

Bühler, K. (1978 [1927]). Die Krise der Psychologie. Stuttgart, Frankfurt/M.: Ullstein.

Bühler, K. (1999 [1934]). Sprachtheorie. Die Darstellungsfunktion der Sprache. Stuttgart: Lucius \& Lucius.

Ebbinghaus, H. (1885). Über das Gedächtnis. Untersuchungen zur Experimentellen Psychologie. Darmstadt: Wissenschaftliche Buchgesellschaft.

Eckardt, G. (2001). Anthropologie und empirische Psychologie um 1800. Ansätze einer Entwicklung zur Wissenschaft. Köln: Böhlau.

Ekman, P. (1992). An Argument for Basic Emotions. cognition and emotion, 6, 169-200.

Hardt, D. (Hrsg.). (1991). Die Erfindung des Gedächtnisses. Texte, zusammengestellt und eingeleitet von Dietrich Hardt. Frankfurt/M.: Keip Verlag.

Herder, J.G. (1778). Vom Erkennen und Empfinden der menschlichen Seele. In J. G. Herder, Werke, Band 4. Hrsg. von J. Brummack u. M. Bollacher (S. 327-393). Frankfurt/M.: Deutscher Klassiker Verlag.

James, W. (1890). The Principles of Psychology. 2 Bände. New York: Dover Publications.

Kandel, E. (2006). Aufder Suche nach dem Gedächtnis. Die Entstehung einer neuen Wissenschaft des Geistes. Münchern: Siedler.

Müsseler, J. \& Prinz, W. (Hrsg.). (2002). Allgemeine Psychologie. Heidelberg, Berlin: Spektrum Akademischer Verlag.

Panksepp, J. \& Biven, L. (2012). The archaeology of Mind. Neuroevolutionary Orignis of Human Emotions. New York, London: Norton.

Pinker, S. (1994/1996). Der Sprachinstinkt. Wie der Geist die Sprache bildet. München: Kindler Verlag.

Rensch, B. (1973). Gedächtnis, Begriffsbildung und Planhandlungen bei Tieren. Berlin: Parey.

Savage-Rumbaugh, E. S., Shanker, S. \& Taylor, T.J. (1998). Apes, language, and the human mind. New York: Oxford University Press.

Schönpflug, W. (2000). Geschichte und Systematik der Psychologie. Ein Lehrbuch für das Grundstudium. Weinheim: Psychologie Verlags Union.

Seidel, R. (2018). Die Evolution der Psyche. Wieviel Tier ist der Mensch? Lengerich: Pabst Science Publishers.

Stearns, P. (1995). Emotion. In R. Harreé \& P. Stearns (Hrsg.), Discursive Psychology in Practice (S. 37-54). London: Sage.

Trommsdorff, G. \& Birbaumer, N. (Hrsg.). (2007). Theorien und Methoden der kulturvergleichenden Psychologie. Göttingen: Hogrefe.

Tulving, E., Szpunar, K. (2009). Episodic memory. Scholarpedia, 4(8), 3332. DOI: 10.4249/scholarpedia. 3332

\section{Der Autor}

Reiner Seidel, Prof. Dr., Dipl.-Psych., war bis 2006 Professor für Allgemeine Psychologie am Fachbereich Erziehungswissenschaften und Psychologie an der FU Berlin. Heute bietet er Lehrveranstaltungen in der Bachelor-Ausbildung an. Derzeitiges Projekt: »Geschichte der Psychotherapie «.

Kontakt: rseidel@zedat.fu-berlin.de 